\title{
Arrival time distribution of muons from extensive air showers
}

\author{
Allan Machado Payeras* and Anderson Campos Fauth \\ University of Campinas, \\ Rua Sérgio Buarque de Holanda 777, Campinas, Brazil \\ E-mail: payeras@ifi.unicamp.br, fauth@unicamp.br
}

\begin{abstract}
The upgraded surface detectors of the Pierre Auger Observatory will provide data that enables the separation of signals due to the muonic component of extensive air showers. Such information permits the application of new event reconstruction methods, that will contribute to composition studies of high-energy cosmic rays and the understanding of their origin. Considering the idea of using the muonic signals from the upgraded surface detectors, we studied the characterisation of muon distributions in extensive air showers using CORSIKA simulations of showers initiated by proton, silicon and iron nuclei with energy of $10^{19} \mathrm{eV}$ and QGSJet-04 as the model of hadronic interaction for high-energies. We analysed the time distribution of muons arriving at the observation level for different radial distances to the shower core. The results were compared with analytical expressions, and agreement was found. The muon time distribution in the observation level was also used to reconstruct the muonic profile in the atmosphere. The understanding of such distributions is crucial for the development of reconstruction methods that can be applied to data from the upgraded Auger Observatory.
\end{abstract}

$37^{\text {th }}$ International Cosmic Ray Conference (ICRC 2021)

July 12th - 23rd, 2021

Online - Berlin, Germany

\footnotetext{
${ }^{*}$ Presenter
} 


\section{Introduction}

The Pierre Auger Observatory [1] was built in Argentina to study cosmic rays with energies above $10^{17} \mathrm{eV}$. The Observatory consists of two sets of independent detectors. The surface detector (SD) covers an area of $3000 \mathrm{~km}^{2}$ with 1660 water-Cherenkov stations disposed in a triangular grid with spacing of $1500 \mathrm{~m}$. The fluorescence detector (FD) comprises 27 fluorescence telescopes distributed in four observation sites around the SD. The SD surveys the particles from extensive air showers (EAS) that reach the observation level, whereas the FD records the longitudinal development of air showers through collection of the emitted fluoresce light.

Although many important results were obtained by the Pierre Auger Collaboration, a better understanding of the astrophysical origin of ultra-high energy cosmic rays demands a larger amount of data sensitive to the composition of the primary particles. For that reason, the Auger Observatory is upgrading its SD stations [2]. The main component of the upgrade is the installation of a scintillator on top of each station. Since the water-Cherenkov detectors and the scintillators have different responses to the muonic and electromagnetic components of EAS, a separation of their signals will be possible. This allows the application of event reconstruction methods that will provide information on the composition of cosmic rays.

We have studied the production of muons in EAS as well as their time distribution in the observation level, which was compared to existing analytical descriptions [3]. A relation between the distribution of production distance in the atmosphere and arrival time was investigated. The main motivation is the prospect of using the muonic signals from the upgraded detectors of the Pierre Auger Observatory to reconstruct events and obtain information related to the composition of the primary particles.

\section{Muon production distributions}

For our studies, we used the CORSIKA software [4] to simulate showers initiated by proton, silicon and iron nuclei with energy of $10^{19} \mathrm{eV}$ and zenith angles of 0,30 and 60 degrees. Hadronic interactions below $80 \mathrm{GeV}$ were treated with FLUKA [5], whereas QGSJet-II-04 [6] was used above that energy. The altitude and magnetic field of the observation level was set to match those of the Pierre Auger Observatory. For each configuration, eight showers were simulated.

In the subsequent analyses, a cylindrical coordinate system was used. The z-axis is defined along the shower axis and the origin of the polar plane is in the point where the z-axis intercepts the observation level. The polar angle $\zeta=0$ is defined in the direction where the shower front first touches the ground. A schematic drawing of this coordinate system is shown in figure 1.

The distribution of muon production distance along the shower axis is shown in figure 2 for primaries of proton with zenith angles of 0,30 and 60 degrees and $10^{19} \mathrm{eV}$. The position of the distribution maximum ${ }^{1}$ increases for higher zenith angles. Since more inclined showers have to traverse a larger path through the atmosphere, their maximum development will occur higher along their axis when compared to showers with lower zenith angle. A fit to a Gaussian function was performed to describe these distributions. The values of the mean $\left(z_{0}\right)$ and standard deviation $\left(\sigma_{z}\right)$,

${ }^{1}$ defined in the z-axis of our coordinate system. 


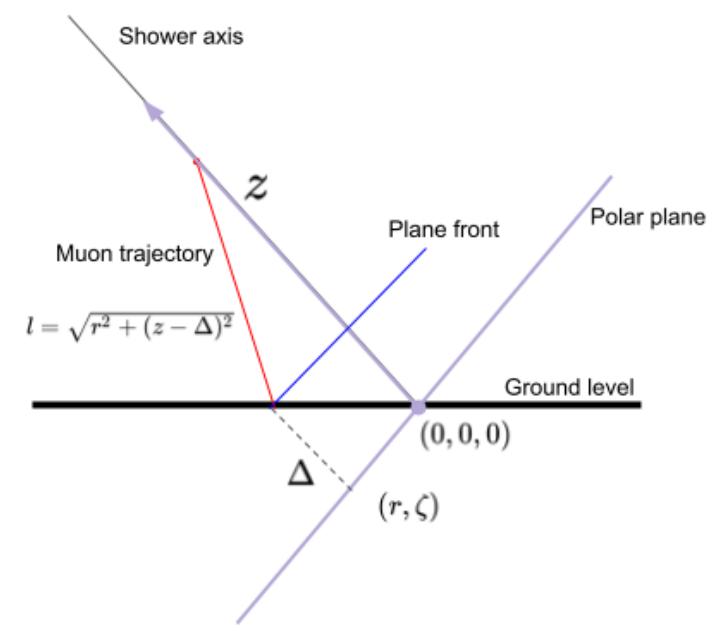

Figure 1: Cylindrical coordinate system used in our analyses. The red line represent the trajectory of a muon produced in the shower.

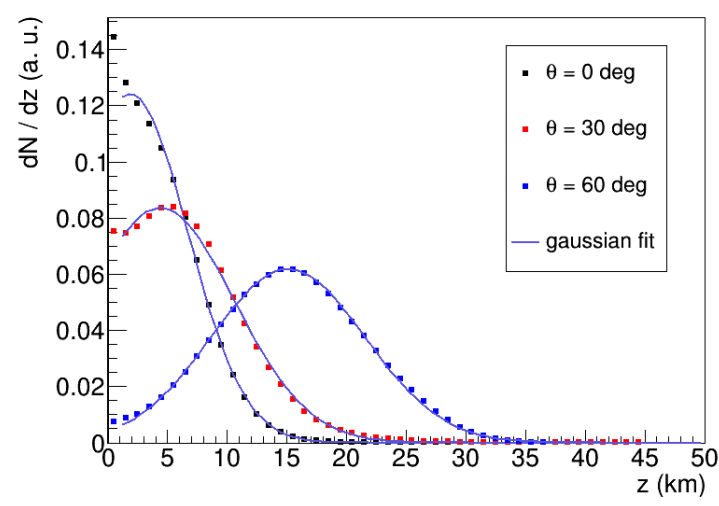

Figure 2: Normalised distribution of production distance of muons along the shower axis for primaries of protons with 0,30 and 60 degrees of zenith angle and energy of $10^{19} \mathrm{eV}$. The distributions were fitted to Gaussian functions.

obtained from the fits, are presented in table 1 for each primary and zenith angle. The values of $z_{0}$ also show that heavier primaries reach their maximum higher in the atmosphere.

The distribution of transverse momentum presented by the muons in the moment of their production is displayed in figure $3 \mathrm{a}$, for primaries of proton with energy of $10^{19} \mathrm{eV}$. The transverse momentum $p_{t}$ is defined as perpendicular to the shower axis. This distribution is due to that of the parent hadrons combined with effects introduced by their decay. We observe that the distribution of transverse momentum is independent of the zenith angle of the primary. To describe the distribution, a fit with the function

$$
f_{p}\left(p_{t}\right)=A p_{t}^{\lambda} \exp \left(-\frac{p_{t}}{Q}\right)
$$

was applied. The values of the parameters $\lambda$ and $Q$, obtained from the fits, can be found in table 1. We observe that these parameters are approximately the same for different primaries and zenith angles. A mean value of $1.317 \pm 0.012$ and $0.12958 \pm 0.00073 \mathrm{GeV} / \mathrm{c}$ were obtained for $\lambda$ and $Q$, respectively.

In figure 3b, the distribution of production energy of muons is shown for showers initiated by primaries of iron nuclei. A weak dependence with zenith angle is observed. The distributions were fitted to a power law

$$
f_{E}(E)=B E^{-\gamma}
$$

We found that the spectral index $\gamma$ do not vary significantly for different primaries and zenith angles, as shown in table 1. A value of $\gamma \sim 2.727 \pm 0.002$ can be taken as a good approximation. This result agrees with that in reference [3].

\section{Arrival time distribution and reconstruction of muonic profile}

For the analysis of the time distribution of muons in the observation level, we have followed the approach described in reference [3]. We also verified the analytical description developed therein 


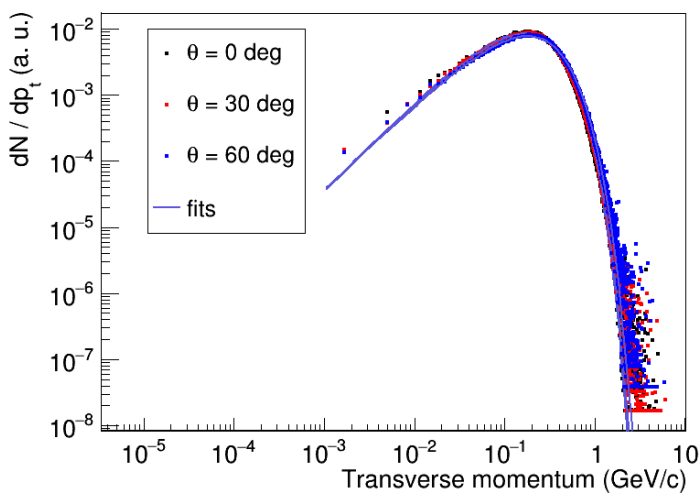

(a)

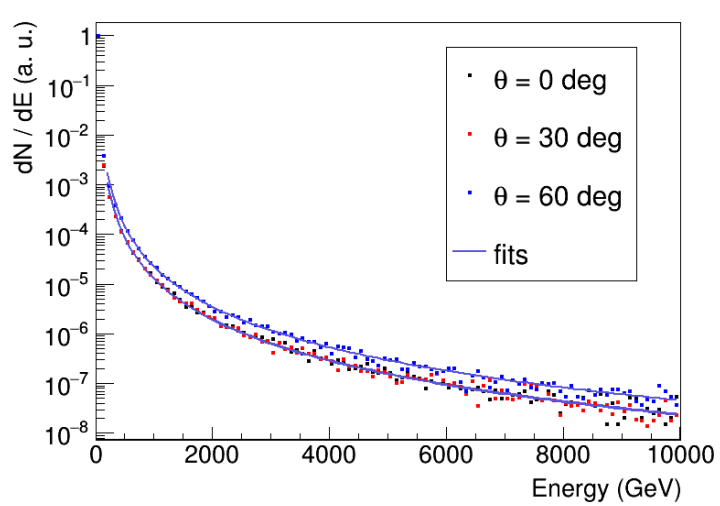

(b)

Figure 3: (a) Normalised distribution of transverse momentum of muons in showers initiated by protons with energy $10^{19} \mathrm{eV}$ and zenith angles of 0, 30, 60 degrees. The distributions were fitted to the function in equation 1. (b) Normalised distribution of production energy of muons for primaries of iron with energy of $10^{19} \mathrm{eV}$ and zenith angles of 0,30 and 60 degrees. A power law was used to fit the distributions.

\begin{tabular}{|c|c|c|c|c|c|c|}
\hline Primary & $\begin{array}{l}\text { Zenith } \\
\text { angle } \\
\text { (deg) }\end{array}$ & $z_{0}(\mathrm{~km})$ & $\sigma_{z}(\mathrm{~km})$ & $\lambda$ & $\mathrm{Q}(\mathrm{GeV} / \mathrm{c})$ & $\gamma$ \\
\hline \multirow{3}{*}{ Proton } & 0 & $\begin{array}{c}1.880 \pm \\
0.010\end{array}$ & $\begin{array}{c}4.7910 \pm \\
0.0048\end{array}$ & $1.34 \pm 0.18$ & $\begin{array}{c}0.123 \pm \\
0.011\end{array}$ & $\begin{array}{c}2.7679 \pm \\
0.0090\end{array}$ \\
\hline & 30 & $\begin{array}{c}4.3654 \pm \\
0.0091\end{array}$ & $\begin{array}{c}6.2210 \pm \\
0.0056\end{array}$ & $1.32 \pm 0.15$ & $\begin{array}{c}0.1275 \pm \\
0.0091\end{array}$ & $\begin{array}{c}2.7540 \pm \\
0.0068 \\
\end{array}$ \\
\hline & 60 & $\begin{array}{c}15.0956 \pm \\
0.0041\end{array}$ & $\begin{array}{c}6.4868 \pm \\
0.0029\end{array}$ & $\begin{array}{c}1.273 \pm \\
0.024\end{array}$ & $\begin{array}{c}0.1410 \pm \\
0.0015\end{array}$ & $\begin{array}{c}2.7541 \pm \\
0.0060\end{array}$ \\
\hline \multirow{3}{*}{ Silicon } & 0 & $\begin{array}{c}1.7158 \pm \\
0.0084\end{array}$ & $\begin{array}{c}4.7538 \pm \\
0.0038\end{array}$ & $\begin{array}{c}1.328 \pm \\
0.028\end{array}$ & $\begin{array}{c}0.1255 \pm \\
0.0016\end{array}$ & $\begin{array}{c}2.7600 \pm \\
0.0070\end{array}$ \\
\hline & 30 & $\begin{array}{c}4.4000 \pm \\
0.0060\end{array}$ & $\begin{array}{c}5.3403 \pm \\
0.0034\end{array}$ & $\begin{array}{c}1.334 \pm \\
0.028\end{array}$ & $\begin{array}{c}0.1267 \pm \\
0.0016\end{array}$ & $\begin{array}{c}2.7329 \pm \\
0.0063\end{array}$ \\
\hline & 60 & $\begin{array}{c}18.9597 \pm \\
0.0047\end{array}$ & $\begin{array}{c}7.6460 \pm \\
0.0042\end{array}$ & $1.26 \pm 0.61$ & $\begin{array}{c}0.1416 \pm \\
0.042\end{array}$ & $\begin{array}{c}2.6842 \pm \\
0.0049\end{array}$ \\
\hline \multirow{3}{*}{ Iron } & 0 & $\begin{array}{c}1.584 \pm \\
0.011\end{array}$ & $\begin{array}{c}5.2966 \pm \\
0.0050\end{array}$ & $\begin{array}{c}1.339 \pm \\
0.028\end{array}$ & $\begin{array}{c}0.1248 \pm \\
0.0016\end{array}$ & $\begin{array}{c}2.7280 \pm \\
0.0064\end{array}$ \\
\hline & 30 & $\begin{array}{c}4.5856 \pm \\
0.0084\end{array}$ & $\begin{array}{c}6.4617 \pm \\
0.0054\end{array}$ & $\begin{array}{c}1.331 \pm \\
0.035\end{array}$ & $\begin{array}{c}0.1278 \pm \\
0.0020\end{array}$ & $\begin{array}{c}2.7487 \pm \\
0.0061\end{array}$ \\
\hline & 60 & $\begin{array}{c}20.1478 \pm \\
0.0046\end{array}$ & $\begin{array}{c}8.0198 \pm \\
0.0041\end{array}$ & $1.26 \pm 0.36$ & $\begin{array}{c}0.142 \pm \\
0.025\end{array}$ & $\begin{array}{c}2.6938 \pm \\
0.0049\end{array}$ \\
\hline
\end{tabular}

Table 1: Parameters obtained from the fits of muon production distributions to the appropriate functions. All primaries have energy of $10^{19} \mathrm{eV}$.

using our simulation data described in section 2. We consider the arrival delay of muons at the observation level with respect to a imaginary plane front that travels along the shower axis at the 
speed of light. In figure 1, this plane front is depicted in blue. Two main effects contribute to the total muon delay: the geometrical and kinematic delays.

If we suppose that muons also travel through the atmosphere with the speed of light, we can verify, with the help of figure 1, that the path traversed by the muon $(l)$ is larger than that travelled by the plane front $(z-\Delta)$. This introduces a geometrical delay in the muon arrival time. Since $l=\sqrt{r^{2}+(z-\Delta)^{2}}$ and $\Delta=r \tan \theta \cos \zeta$, where $\theta$ is the zenith angle and $(r, \zeta)$ the muon coordinates in the polar plane, the geometrical delay is given by

$$
t_{g}=\frac{1}{c}\left[\sqrt{r^{2}+(z-\Delta)^{2}}-(z-\Delta)\right],
$$

where $c$ is the speed of light in vacuum. The distribution of geometrical delay for the muons in the observation level is given by

$$
\begin{aligned}
g\left(t_{g}\right) & =\frac{d N}{d t_{g}} \equiv-\frac{1}{N_{r}} \frac{d^{2} N}{d t_{g} d r} \\
& =-h(z) \frac{d z}{d t_{g}} \frac{1}{N_{r}} \int \frac{d^{2} N}{d E d r}[z] d E,
\end{aligned}
$$

where $N_{r}$ is short notation for $d N / d r$ and $h(z)$ is the distribution of production distance which, as showed in section 2, can be approximated with a Gaussian function. The factor $d z / d t_{g}$ can be easily obtained from equation 3. An analytical expression for the radial energy distribution in the integral is given in reference [3].

Since muons travel at subluminal speeds, a delay is introduced in relation to a particle travelling at the speed of light. This is called the kinematic delay. Using a model for the energy loss of muons in their path through the atmosphere [3], the kinematic delay can be written as

$$
t_{\epsilon}=\frac{1}{2} \frac{\left(m c^{2}\right)^{2}}{c \rho k}\left[\frac{1}{E_{i}-\rho k l}-\frac{1}{E_{i}}\right]
$$

where $m$ is the muon mass, $E_{i}$ is the muon energy at the moment of its production, $\rho$ is the atmosphere density and $k$ is the muon energy loss per crossed distance. For our analysis we used $\rho=0.001 \mathrm{~g} \mathrm{~cm}^{-3}$ and $k=2 \mathrm{MeV} / \mathrm{g} \mathrm{cm}^{-2}$. The distribution of kinematic delay of the muons in the observation level is

$$
\begin{aligned}
\epsilon\left(t_{g}\right) & =\frac{d N}{d t_{\epsilon}} \equiv-\frac{1}{N_{r}} \frac{d^{2} N}{d t_{\epsilon} d r} \\
& =-\frac{1}{N_{r}} \frac{d^{2} N}{d E_{i} d r} \frac{d E_{i}}{d t_{\epsilon}},
\end{aligned}
$$

where the factor $d E_{i} / d t_{\epsilon}$ can be derived using equation 5 .

The muon total delay with respect to the plane front is $t_{g}+t_{\epsilon}$. Therefore, the total delay distribution is given by the convolution of the distributions of the geometrical and kinematic delays

$$
\frac{d N}{d t}=g(t) \otimes \epsilon(t)=\int g\left(t-t^{\prime}\right) \epsilon\left(t^{\prime}\right) d t^{\prime} .
$$

In figure 4, we show the distribution of muon delay at different distances from the shower axis, as obtained from the CORSIKA simulations. Plots $4 \mathrm{a}$ and $4 \mathrm{~b}$ are for a primary of proton, $4 \mathrm{c}$ and 


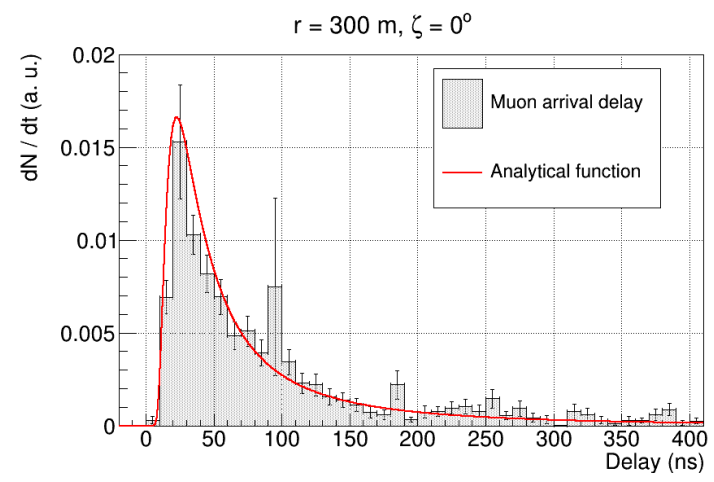

(a)

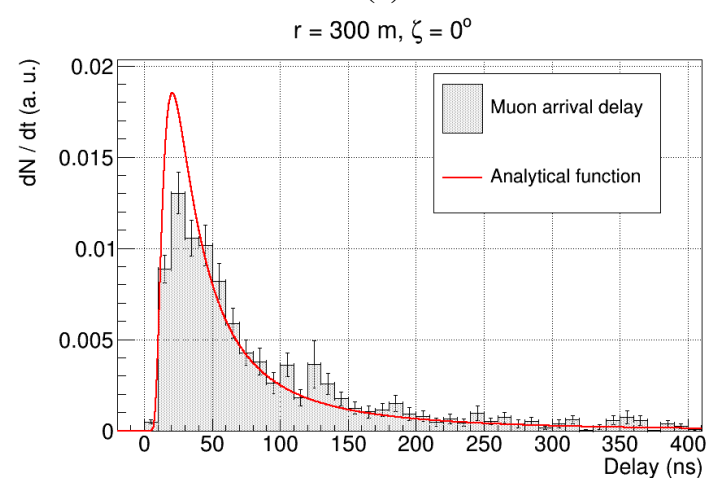

(c)

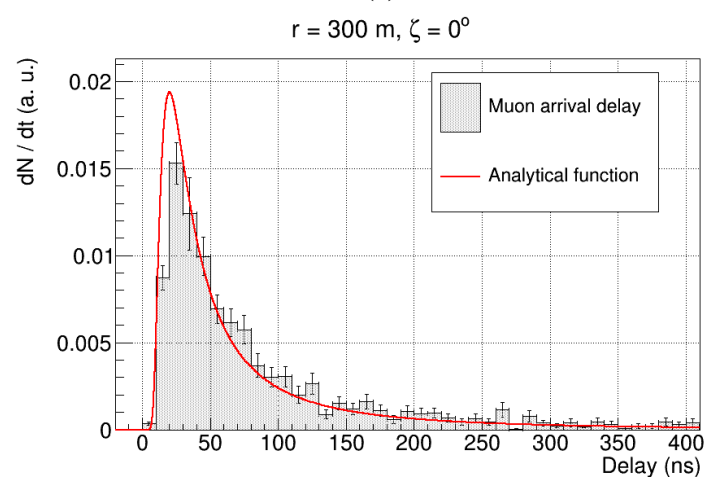

(e)

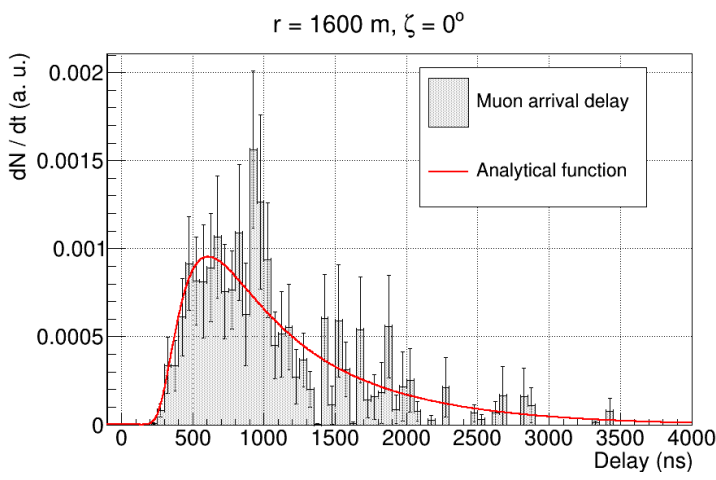

(b)

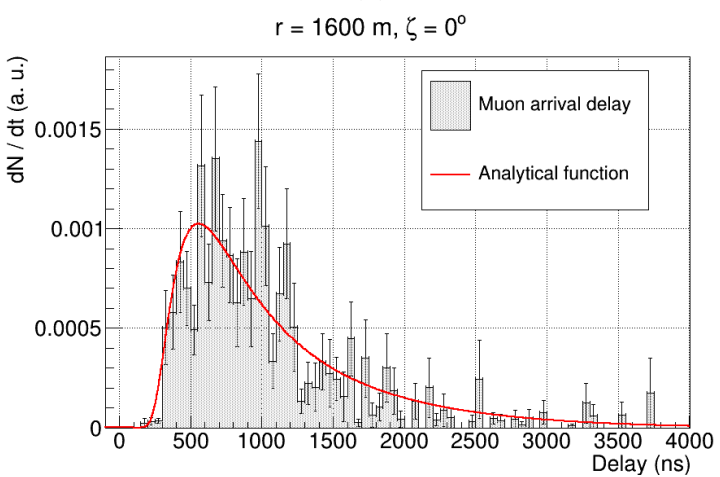

(d)

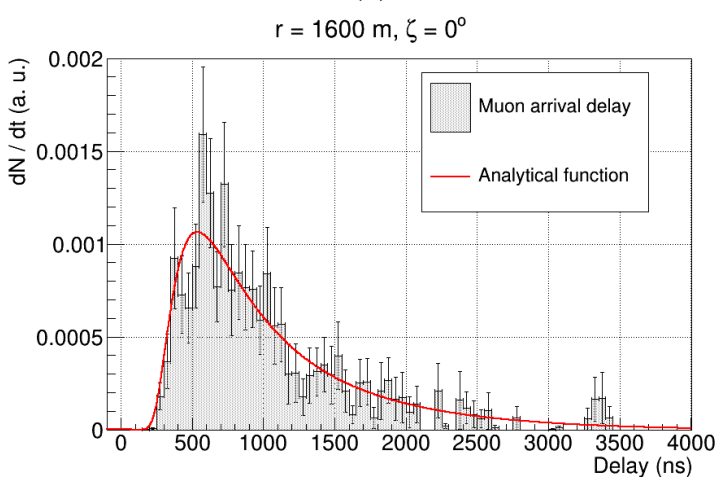

(f)

Figure 4: Normalised distributions of muon arrival delay. The distributions in the left column are for muons arriving at $300 \mathrm{~m}$ from the shower axis and $\zeta=0 \mathrm{deg}$, whereas in the right column $(r, \zeta)=(1600 \mathrm{~m}, 0 \mathrm{deg})$. Plots (a) and (b) are for a proton primary, (c) and (d) silicon and (e) and (f) for iron. All primaries have zenith angle of 30 degrees and energy of $10^{19} \mathrm{eV}$.

$4 \mathrm{~d}$ for silicon and $4 \mathrm{e}$ and $4 \mathrm{f}$ for iron. All primaries have zenith angle of 30 degrees and energy of $10^{19} \mathrm{eV}$. The red lines indicate the analytical delay distribution obtained from the convolution of the geometrical and kinematic delay distributions (equation 7). We verify that the analytical function describes very well the delay distribution for different primaries and distances to the shower axis.

For distances above $1000 \mathrm{~m}$ from the shower core the kinematic delay can be neglected when compared to the geometrical delay $[3,7]$. The muon total delay distribution at $(r, \zeta)=$ 


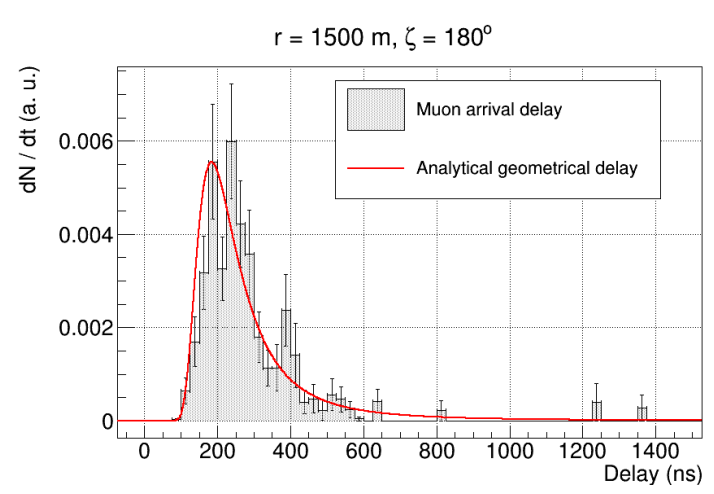

(a)

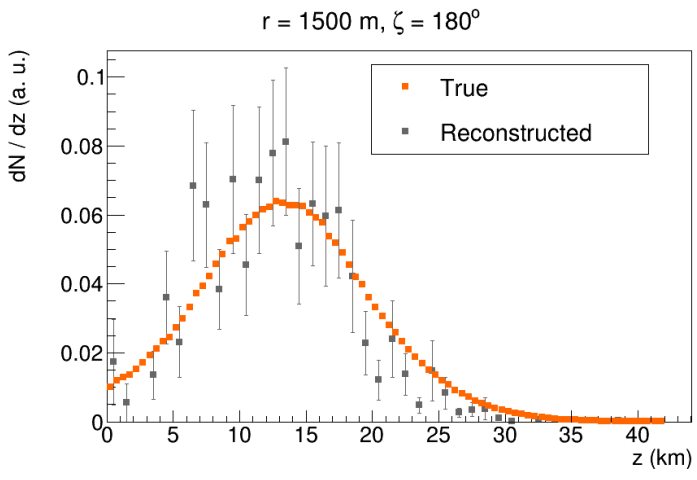

(b)

Figure 5: (a) Normalised total muon delay distribution at $(r, \zeta)=(1500 \mathrm{~m}, 180 \mathrm{deg})$ for a primary of proton with zenith angle of 60 degrees and $10^{19} \mathrm{eV}$. The red line shows the analytical geometrical delay distribution. (b) Reconstructed and true distribution of muon production distance for the same event.

$(1500 \mathrm{~m}, 180 \mathrm{deg})$ is shown in figure 5 a for a primary of proton with zenith angle of 60 degrees and energy of $10^{19} \mathrm{eV}$. The red line shows the predicted distribution using only the geometrical delay which, in this case, works as a good approximation of the total delay distribution.

Since the geometrical delay is related to the distribution of production distance, as seen in equation 4 , one can use the observed delay distribution to reconstruct the profile of muon production in the atmosphere. This is interesting because the composition of the primary particle is related to the point at which the maximum production of muons occurs during the shower development.

To reconstruct the muonic profile, we first convert the observed delay distribution of muons into a distribution of production distance, using equation 3. Next, this distribution is divided by the functions $-d z / d t_{g}$ and $\int\left(d^{2} N / d E d r\right) d E$ (see eq. 4). Finally, we normalise the resulting distribution to obtain the corresponding normalised distribution of production distance of the muons.

Using the delay distribution of figure 5a, we reconstructed the muonic distribution of production distance. The result is presented in figure $5 \mathrm{~b}$. We observe that the reconstruction procedure is consistent with the true production distance distribution, represented by the orange points.

In view of the upgraded surface detector of the Pierre Auger Observatory, and its potential for disentangling the muonic signals, a method can be developed to use the new data for reconstructing the muonic profile of showers and, therefore, provide a means to study the composition of the primaries.

\section{Summary}

In this work, we used the software CORSIKA to simulate extensive air showers initiated by protons, silicon and iron nuclei with zenith angles of 0,30 and 60 degrees and energy of $10^{19} \mathrm{eV}$. We studied the muon distributions of production distance, transverse momentum and energy. Fits of these distributions to functions were performed and the corresponding parameters were obtained.

Using the simulation data, we studied the distribution of arrival delay presented by muons in the observation level for different primaries and distances to the shower core. We verified that the 
analytical description of these distributions in terms of the geometrical and kinematic delays is consistent with the simulation data.

Finally, the muon time distribution in the observation level was used to reconstruct the profile of muonic production in the atmosphere. A consistent result was obtained which supports the possibility of using data of the upgraded surface detectors of the Pierre Auger Observatory for reconstruction of muonic profiles. This will allow composition studies and ultimately help in the understanding of the nature of ultra-high energy cosmic rays.

\section{Acknowledgments}

The authors thank the financial support from the São Paulo Research Foundation (FAPESP), grants \#2019/15899-5 and \#2019/10151-2. This study was also financed in part by the Coordenação de Aperfeiçoamento de Pessoal de Nível Superior - Brasil (CAPES) - Finance Code 001.

\section{References}

[1] A. Aab et al., The Pierre Auger cosmic ray observatory, Nucl. Instrum. Methods Phys. Res., Sect. A 798 (2015) 172 .

[2] A. Aab et al., The Pierre Auger observatory upgrade - preliminary design report, arXiv:1604.03637 (2016) .

[3] L. Cazón, R. Vázquez, A. Watson and E. Zas, Time structure of muonic showers, Astropart. Phys. 21 (2004) 71.

[4] D. Heck, J. Knapp, J. Capdevielle, G. Schatz and T. Thouw, CORSIKA: A Monte Carlo code to simulate extensive air showers. Forschungszentrum Karlsruhe, fzka-6019 ed., 1998.

[5] A. Ferrari, P.R. Sala, A. Fassò and J. Ranft, FLUKA: A multi-particle transport code (program version 2005), CERN Yellow Reports: Monographs, CERN, Geneva (2005), 10.5170/CERN-2005-010.

[6] S. Ostapchenko, Monte carlo treatment of hadronic interactions in enhanced pomeron scheme: Qgsjet-ii model, Phys. Rev. D 83 (2011) 014018.

[7] L. Cazon, R. Conceição, M. Pimenta and E. Santos, A model for the transport of muons in extensive air showers, Astropart. Phys. 36 (2012) 211. 